\title{
Voir Neptune au bout de sa plume
}

Flammarion et la constitution de l'algèbre comme nouvel instrument de vision

Yohann Ringuedé

\section{(2) OpenEdition}

Journals

Édition électronique

URL : http://journals.openedition.org/aes/1012

DOI : 10.4000/aes.1012

ISSN : 2258-093X

Éditeur

Laboratoire LISAA

Référence électronique

Yohann Ringuedé, "Voir Neptune au bout de sa plume », Arts et Savoirs [En ligne], 8| 2017, mis en ligne le 19 avril 2017, consulté le 24 avril 2019. URL : http://journals.openedition.org/aes/1012 ; DOI : 10.4000/aes.1012

Ce document a été généré automatiquement le 24 avril 2019

Centre de recherche LISAA (Littératures SAvoirs et Arts) 


\section{Voir Neptune au bout de sa plume}

Flammarion et la constitution de l'algèbre comme nouvel instrument de vision

Yohann Ringuedé

C'est toi, Planète en ces lointains perdue,

Toi qui fermant ce fuyant horizon,

De nos confins sentinelle assidue,

Erras longtemps invisible et sans nom, Jusqu'au moment où l'algèbre idéale, De son œil pâle et de sa main fatale,

Vint te saisir au fond de ton désert

Et te rendit au céleste concert. ${ }^{1}$

M. Le Verrier a aperçu le nouvel astre sans avoir besoin de jeter un seul regard vers le ciel ; il l'a vu au bout de sa plume [...]. ${ }^{2}$

1 Une grande partie de la recherche astronomique de la première moitié $\mathrm{du} \mathrm{XIX}^{\mathrm{e}}$ siècle gravite autour d'un problème immense: les tables qui décrivent les mouvements des planètes révèlent une anomalie touchant la dernière d'entre elle. En effet, les astronomes calculent alors l'allure des planètes connues, tout au long de leur révolution orbitale, en croisant les observations effectuées et les connaissances newtoniennes de la mécanique céleste. Ils doivent pour cela tenir compte de la puissance d'attraction qu'exerce le soleil sur la course de chacune de ces planètes, mais aussi et surtout, des attractions collatérales qu'exercent par intermittence les planètes les unes sur les autres. Uranus, découverte un peu par hasard en 1781 par Herschel qui croyait d'abord n'avoir sous les yeux qu'une comète, voyage dans l'espace selon un train erratique et incompréhensible. Tous les astronomes le constatent, tous s'en étonnent. Il y a là, certes, un mystère propre à constituer des spéculations dignes des fictions les plus palpitantes : sous ce voile se cache quelque chose à voir. À partir des années trente se répand l'idée selon laquelle une planète inconnue, alors nommée "planète troublante ", pourrait, au-delà de l'orbite elliptique d'Uranus, exercer une force gravitationnelle qui justifierait les variations de vitesse de cette dernière planète connue ${ }^{3}$. 
2 Le cas est tout à fait nouveau dans l'histoire des sciences et de la découverte scientifique. L'existence d'un objet céleste d'importance majeure est formulée hypothétiquement selon la structure mathématique d'une pure équation à inconnues multiples. Il faut dès lors résoudre un problème que les mathématiques appellent inverse, c'est-à-dire un problème à propos duquel l'inconnue n'est appréhendable que selon les effets qu'elle produit. Cette planète troublante (terme qui se charge d'une syllepse lourde de sens), n'est pour l'instant appréhendée que par hypothèse, en fonction de l'attraction supposée qu'elle exercerait sur Uranus. Sans cette attraction, dont on constate les effets mais dont on ne peut que conjecturer la source, l'allure irrégulière de la planète d'Herschel est inexplicable. Les astronomes sont en présence d'une façon toute nouvelle d'appréhender la découverte scientifique: Herschel aperçoit Uranus par la pure observation télescopique, alors que la découverte par Urbain Le Verrier de la "planète troublante " s'opérera par la seule résolution algébrique en 1846. Cet événement scientifique fait basculer l'astronomie tout entière dans une nouvelle approche du ciel: à la lunette astronomique vient se substituer le nombre comme seul outil capable de découvrir de nouveaux objets célestes ${ }^{4}$.

3 La découverte de Neptune eut un impact sensationnel dans le monde scientifique et dans le public. Elle influence par conséquent certaines entreprises littéraires qui ont partie liée avec la science : certains romans ${ }^{5}$, des poèmes astronomiques ${ }^{6}$, mais aussi les grands ouvrages des vulgarisateurs. Se met en place un nouveau savoir voir (algébrique et non plus empirique) dont l'impact semble déterminant. Camille Flammarion, le plus réputé et le plus populaire des astronomes à la fin du siècle, y consacre un chapitre conséquent de son Astronomie populaire, dont la première édition paraît en $1880^{7}$.

Danielle Chaperon l'a bien montré : Flammarion est un homme qui cherche à «faire voir » le cosmos. Telle est sa "technique » ${ }^{8}$. Lorsque le média qu'il choisit est textuel, la "machinerie » optique est prise en charge par l'«écriture »". De fait, Flammarion se proclame à la fois astronome et « littérateur $»^{10}$. Le terme est axiologiquement marqué : il ne se dit pas écrivain. La diffusion scientifique prend appui chez lui sur les outils rhétoriques de la littérature. La conception est assurément " utilitaire », pour reprendre le terme de Danielle Chaperon ; il n'empêche que Flammarion (qui suit en cela le précepte comtien selon lequel le poète doit être au service de la science) travaille la langue en vue d'un but précis, celui de la diffusion. Certes, à l'heure de l'autonomisation du fait littéraire, la conception ancillaire qu'il se fait des lettres incite la postérité à considérer l'entreprise du savant lettré comme anachronique. C'est oublier que le second versant du siècle est marqué par la coprésence agonistique de deux formes de modernités littéraires mises en scène par Antoine Compagnon dans ses Cinq paradoxes de la modernité : face aux tenants de la forme pure et de l'art pour l'art, une autre conception de la littérature moderne, portée notamment par Maxime Du Camp, exige qu'elle rende hommage aux innovations techniques et aux sciences ${ }^{11}$. Il faut parier pour ce faire sur la rénovation thématique et sur le retour à un rôle pragmatique, voire propédeutique, des écrivains et des poètes ${ }^{12}$. Flammarion s'inscrit dans ce courant. Comme l'écrit justement Danielle Chaperon, la littérature selon lui « est vouée à une disparition prochaine si elle ne se met pas tout entière au service de la diffusion des savoirs $»^{13}$. Ainsi, la tentative de l'astronome peut et doit être appréhendée à l'aune du projet qui est le sien. Cette entreprise est double: le savant utilise des outils littéraires pour communiquer son savoir, mais en retour, la littérature puise dans la science de quoi la sauver d'une mort prochaine. 
5 Dans le cadre d'un tel projet, l'histoire de la découverte de Neptune telle qu'elle est relatée, une trentaine d'années après sa révélation, dans l'Astronomie populaire ${ }^{14}$, est particulièrement emblématique de ce que science et littérature peuvent mutuellement s'apporter. Comme l'écrit Danielle Chaperon: "L'acte vulgarisateur est une communication "dramatique", avec ses personnages, son intrigue, ses péripéties, son dénouement. $»^{15}$ Cette « communication » dramatique met donc en scène une histoire probante, mais cette histoire n'est pas celle que l'on attend. Flammarion, en effet, relate dans un premier temps l'histoire de la révélation de Neptune, mais il diffère le "dénouement», dans le but de mettre en débat la constitution tout algébrique de l'astronomie moderne. Ainsi Flammarion met-il les ressources de la littérature au profit de l'anecdote strictement scientifique et de l'éloge de Le Verrier, ce qui constituera le premier point de cette étude. Néanmoins, la figure de Le Verrier, telle que la dépeint Flammarion, n'est pas axiologiquement univoque: la description tout élogieuse du mathématicien en savant romantique et prophète (ce qui, en soi, constitue déjà un paradoxe) alterne avec celle d'un scientifique obtus et frappé de myopie. Cette ambiguïté est indiquée pour, dans un second temps du chapitre, permettre à Flammarion de mettre en place sa propre vision de la nouvelle méthode astronomique, qui, assurément, repose sur l'algèbre, mais ne vise pas le nombre comme une fin en soi : l'astronomie à venir, selon lui, doit compléter les données du nombre par un travail de l'imagination.

6 La forme du texte de Flammarion diverge de celle d'Arago qui relate la même découverte dans sa propre Astronomie populaire ${ }^{16}$ : le directeur de l'observatoire de Paris donne à ses cours, réunis de façon posthume, l'allure de mémoires. Flammarion y ajoute le sel de la narration. Le texte du chapitre IX, sur Neptune, s'ouvre en effet par un petit résumé de sa découverte. En quinze lignes à peine, l'affaire est dévoilée :

On a dit avec raison que les travaux de l'astronomie sont ceux qui donnent la plus haute mesure des facultés de l'esprit humain. La découverte de Neptune, due à la seule puissance des nombres, est l'un des plus éloquents témoignages de cette vérité. L'existence de cette planète dans le ciel a été révélée par les mathématiques. Ce monde, éloigné à plus d'un milliard de lieues de notre station terrestre, est absolument invisible à l'œil nu. Les perturbations manifestées par le mouvement de la planète Uranus ont permis de dire au mathématicien: la cause de ces perturbations est une planète inconnue, qui gravite au-delà d'Uranus, vers telle distance, et qui, pour produire l'effet observé, doit se trouver actuellement en tel point du ciel étoilé. On dirige une lunette vers le point indiqué, on cherche l'inconnue, et, en moins d'une heure, on l'y trouve !17

7 La rhétorique de ce bref paragraphe en forme de captatio benevolentiae est caractérisée par la miniaturisation et l'allusion. Le problème dont il va être question est marqué du sceau de l'exceptionnel et de l'apologue : cette découverte est un « éloquent [...] témoignage ». Les détails sont passés sous silence: les formulations verbales passives, les pronoms indéfinis, l'utilisation du titre (le «mathématicien ») plutôt que du nom propre, tiennent dans le contre-jour l'agent de la découverte et les actions des scientifiques. Cette captatio laisse planer une ombre et suscite la pulsion de vision sous laquelle se cache le désir de savoir. Puisque le voir permet l'accès au savoir, le jour doit être porté sur cette histoire encore marquée par l'obscurité : voilà donc un paragraphe programmatique qui justifie à lui seul toute la démarche vulgarisatrice de Flammarion.

8 L'écrivain rend assez exactement compte de la découverte qui repose sur le modèle de l'équation à inconnues multiples. Après le paragraphe introductif, le texte se poursuit par 
une situation initiale relativement apaisée, qui correspond à une loi mathématique prenant la forme d'une adéquation :

Les astronomes construisent d'avance les tables des positions des planètes dans le ciel [...]. Un astronome de Paris, Bouvard, calculant, en 1820, les tables de Jupiter, Saturne et Uranus, constata que les positions théoriques données par ses tables s'accordaient parfaitement avec les observations modernes pour les deux premières planètes. ${ }^{18}$

Il s'agit d'un état marqué par l'équilibre entre le monde et sa représentation, un « accord [...] parfait » entre deux données de nature différente : la théorie et l'observation. La trajectoire observable, dans un premier temps, reflète exactement la prédiction théorique. Or, la suite directe de la même phrase introduit à la fois un élément perturbateur (en termes narratologiques) et une inconnue qui vient déstabiliser l'adéquation: «[...] tandis que pour Uranus, il y avait des différences inexplicables $»^{19}$. Voilà un état déséquilibré de la relation entre la prédiction théorique et l'observation. L'observation de l'ellipse d'Uranus ne correspond plus à la théorie newtonienne à cause d'éléments perturbateurs pour l'instant indéfinis, des inconnues. L'adéquation prend alors la forme d'une équation.

Le «problème » qui « était à l'ordre du jour $»^{20}$ est ainsi défini, et Arago le soumet au jeune Le Verrier. Or, le texte ne le dit pas ouvertement, mais ce problème est à inconnues multiples : il comporte une grande quantité d'éléments indéterminés combinés. Selon les historiens des sciences ${ }^{21}$, c'est une équation qui en comporte quatorze. L'observation doit donc s'enrichir de l'éclaircissement de quatorze zones d'ombre pour concorder à nouveau avec la prédiction théorique. Il faut ajouter à l'observation des planètes des éléments encore inconnus pour que ce que l'on voit du ciel coïncide à nouveau avec ce que l'on sait de la mécanique céleste. Ces éclaircissements concernent la caractérisation de cette planète troublante dont le nom prend ici tout son sens. On le voit, cette inadéquation qui constitue l'élément perturbateur de la diégèse, représente un " magnifique problème $»^{22}$ $\mathrm{du}$ fait de son ampleur. Les inconnues sont plus nombreuses que les certitudes.

11 Les calculs qu'entreprend Le Verrier en vue de la résolution de cette équation correspondent à l'action de l'anecdote scientifique relatée: «le jeune savant se mit à l'œuvre immédiatement $»^{23}$. Or, comme Le Verrier a affaire à une équation complexe, l'action mathématique est marquée littérairement par l'itération. Tout d'abord, le mathématicien s'attelle à la vérification des seules certitudes que comporte le problème :

Il commença par vérifier les tables de Bouvard, dans lesquelles il corrigea plusieurs erreurs; mais ces erreurs ne rendaient pas compte de la différence trouvée. Recommençant tout le calcul des perturbations de Saturne sur Uranus, il y ajouta celles de Jupiter, recalcula l'orbite d'Uranus d'après les 19 observations anciennes des positions de la planète observée comme étoile avant 1781 et les 179 observations faites de 1781 à 1845 , et vérifia que l'écart entre les positions observées et les positions calculées ne pourrait pas être expliqué par les perturbations de Saturne et de Jupiter. ${ }^{24}$

12 En somme, la multiplicité des opérations décrites ici, marquées significativement par l'itération (que l'on pense au préfixe re-, utilisé deux fois, mais également au sémantisme même du verbe vérifier), a pour l'instant pour premier but d'analyser et d'affiner la valeur des théories validées par l'usage et le temps. Il faut tout d'abord vérifier que le problème existe bien (des erreurs de calcul auraient pu entraîner cette inadéquation), mais aussi s'assurer de la validité des bases qui constitueront les seules certitudes dans la résolution 
du problème. À l'issue de cette première salve d'opérations, Le Verrier, par le biais d'un discours rapporté qui dynamise le récit, le dit lui-même :

«J'ai démontré, dit-il, qu'il y a incompatibilité formelle entre les observations d'Uranus et l'hypothèse que cette planète ne serait soumise qu'aux actions du Soleil et des autres planètes agissant conformément au principe de la gravitation universelle. On ne parviendra jamais, dans cette hypothèse, à représenter les mouvements observés. $»^{25}$

Il s'agit donc, dans ce premier temps de la démarche algébrique, de vérifier et de déterminer les valeurs les plus exactes possibles des données issues de l'observation. La seule théorie stable en laquelle le jeune savant a une confiance aveugle est celle de la mécanique newtonienne :

En présence de cette incompatibilité bien démontrée, Le Verrier ne doute pas un seul instant de l'exactitude de la loi de la gravitation universelle; il rappelle qu'à plusieurs reprises, pour expliquer des inégalités dont on n'avait pu se rendre compte, on s'en est pris à cette loi, qui est toujours sortie victorieuse après un examen plus approfondi des faits. ${ }^{26}$

En effet, l'une des deux explications possibles du voyage erratique d'Uranus était bien le manque de rigueur de la loi de Newton. Le Verrier tranche : c'est une loi qui repose sur un argument d'expérience ; elle fait autorité. La base théorique de l'attraction newtonienne ne doit pas être remise en question. Le problème est alors solidement établi, et l'inconnue majeure à déterminer est hypothétiquement (et héroïquement) définie: "Il aborde hardiment l'hypothèse d'une planète agissant d'une manière continue sur Uranus $»^{27}$. Le grand $X$ de cette équation, que le problème algébrique divise en quatorze petites inconnues, est donc une planète à découvrir. La monstrueuse formule algébrique à quatorze inconnues doit être affrontée... Le Verrier, rompu aux calculs à inconnues multiples (« Déjà accoutumé aux difficultés du calcul des perturbations par ses recherches sur les comètes $»^{28}$ ), connaît la seule solution viable en ce cas : il est nécessaire, considère la recherche algébrique, de déterminer aléatoirement certaines des inconnues en vue de simplifier le problème. Voilà donc une nouvelle étape de la démonstration mathématique qui repose sur la supposition :

Le fait de l'existence d'une planète extérieure étant désormais certain, il suppose, d'après la série de Titius exposée plus haut, que cette planète doit être à la distance 36 et par conséquent graviter en 217 années autour du Soleil, et, dans cette hypothèse, il calcule quelle position elle a dû avoir dans le ciel, derrière Uranus, pour produire par son attraction les écarts observés, et quelle doit être sa masse pour expliquer la grandeur de sa déviation..$^{29}$

Deux inconnues sont ainsi supposées. La loi de Titius-Bode, qui observe empiriquement un rapport entre les distances des planètes connues par rapport au soleil ${ }^{30}$, permet d'imaginer, en la prolongeant, la distance probable de la nouvelle planète par rapport au soleil. Cette supposition lui permet de déduire mathématiquement une hypothétique durée orbitale. À ce stade de la démonstration, deux inconnues sont résolues. La seconde supposition est formulée incidemment à travers l'expression " derrière Uranus » : pour simplifier son calcul, Le Verrier conjecture que le plan de la révolution elliptique de sa planète troublante est le même que celui d'Uranus. Le fil algébrique se dénoue ici, et cette seconde hypothèse de travail permet au mathématicien de résoudre encore deux nouvelles inconnues.

La formule est ainsi simplifiée, ce qui permet à Le Verrier de l'éprouver en recalculant l'orbite d'Uranus : «[il] trouve que toutes les positions s'accordent avec la théorie » ${ }^{31}$. En 
somme, l'équation est redevenue une adéquation, car toutes les inconnues ont été supposées ou déterminées. Flammarion souligne la symétrie entre l'équilibre de la situation initiale, et celui de la situation finale, après résolution du déséquilibre. Dans les deux cas, le calcul et les observations concordent, ce que souligne le polyptote appliqué au verbe accorder. Ainsi, le texte "les positions théoriques données par les tables s'accordaient parfaitement avec les observations modernes ", devient après résolution mathématique du problème : «les positions s'accordent avec la théorie ». La première formule, à l'imparfait, renvoie cet état initial dans un passé pré-algébrique de l'histoire des sciences. Dans la seconde, le pur calcul mathématique a retrouvé dans le présent de l'énonciation un état pré-critique de la relation entre la théorie et l'observation, en même temps que la résolution du nœud narratologique revient à la situation initiale apaisée : « Dès lors, le problème était résolu. ॥"

Il est notable que Flammarion décrive si rapidement la résolution du problème alors qu'il multiplie les formules itératives pour indiquer le caractère laborieux du travail de préparation qui vise à vérifier et fixer la formule de l'équation. Les historiens des sciences indiquent tout de même que le calcul de Le Verrier fut extrêmement difficile, et certains évoquent un délai (certes rapide, dans l'absolu) de trois mois pour calculer l'orbite et la masse de la planète troublante. L'opération, sous la plume de Flammarion, semble frappée du sceau de l'évidence lumineuse et de l'immédiateté. Le Verrier parait n'avoir jamais tâtonné et toujours su exactement où le menait sa démonstration. Le calcul résolu livre alors des chiffres qui permettent de désigner, à un point précis du ciel, l'emplacement de l'objet non identifié : « le 31 août 1846, Le Verrier annonça à l'Académie des sciences que la planète devait se trouver à la longitude $326^{\circ}$, ce qui la plaçait à $5^{\circ}$ à l'est de l'étoile $\delta$ du Capricorne. $\|^{33} \mathrm{~A}$ l'annonce modalisée de la situation de cette planète répond une parole à la pragmatique performative. Le verbe ici crée l'objet céleste en le désignant et par là le donne à voir au regard humain. Cette immédiateté ouvre la voie à une caractérisation toute démiurgique de Le Verrier et des chiffres qu'il manipule. Ils jettent une lumière éclatante et immédiate sur un nouvel objet céleste, la plume du calculateur écrit fiat lux : "Voilà l'histoire de la découverte de Neptune dans sa simple grandeur " ${ }^{34}$. Et les myopes astronomes n'ont plus qu'à constater qu'ils ont désormais, grâce au chiffre, "les yeux ouverts ». Le texte de Flammarion repose donc sur une mise en récit dramatique de cet événement dans l'histoire astronomique, au cours duquel Le Verrier a « vu au bout de sa plume » une nouvelle planète, pour reprendre l'expression d'Arago.

Les protagonistes de cette aventure se voient donc héroïsés: Arago, notamment, est le modèle du savant en avance sur son temps: «Ce problème était à l'ordre du jour, et Arago, toujours à l'avant-garde du progrès, conseilla à un jeune et habile calculateur [...] de résoudre ce magnifique problème. ${ }^{35}$ Arago orchestre la découverte comme s'il en connaissait déjà la partition. Il y a de la magie dans cet épisode, du sublime dans ce «magnifique problème». De même, la jeunesse de Le Verrier est martelée à maintes reprises et considérée comme contradictoire avec la grandeur de la découverte qu'il effectue. Cette héroïsation se double dramatiquement d'incroyables concours de circonstances : «[...] Bessel lui-même commençait la recherche mathématique quand il fut frappé de la maladie qui devait l'emporter au tombeau. » L'idée se fait jour qu'une funeste malédiction touche quiconque s'attelle à ce problème. Du texte se dégage alors à la fois un grand mystère et un soupçon d'élection : tout concourt à faire place libre à Le Verrier. Bessel meurt inopinément ; Arago, figure tutélaire s'il en est, choisit le « jeune » 
mais « habile » Le Verrier, tandis qu'un autre concours de circonstance avait empêché un astronome anglais d'entériner cette découverte :

[...] ce qui va nous surprendre encore davantage, c'est de savoir que, près d'un an auparavant, en octobre 1845, un jeune étudiant de l'université de Cambridge, M. Adams, avait cherché la solution du même problème, obtenu les mêmes résultats, et communiqué ces résultats au directeur de l'observatoire de Greenwich, sans que l'astronome auquel ces résultats étaient confiés en eût rien dit [...]. ${ }^{36}$

La présence du thème de la surprise est éloquente: tout se passe comme si cette découverte était réservée à un jeune élu, étranger à l'observatoire, qui doit en faire une « révélation ». Le mot, significativement, est répété trois fois. Le Verrier devient sous la plume de Flammarion un oracle au sens littéral, un mage délivrant aux astronomes un message qui leur est voilé : « ce n'est qu'après la découverte visuelle de Neptune qu'ils [les astronomes] eurent les yeux ouverts et sentirent un instant le vertige de l'infini devant l'horizon révélé par la perspective neptunienne. $\aleph^{37}$ C'est pourquoi Le Verrier est un «transcendant mathématicien $~_{38}$. Flammarion, à l'occasion d'une citation de Schiller à propos de Christophe Colomb, en vient donc à qualifier Le Verrier de " génie » : « Il y a là, [...] l'expression d'une des conditions les plus réelles du vrai génie dans les sciences, à qui les découvertes n'arrivent point par un hasard, mais qui va au-devant d'elle par une sorte de pressentiment. $»^{39}$ La sublime supériorité de Le Verrier est telle qu'il ne vérifie même pas empiriquement les résultats de ses équations mathématiques :

L'auteur du calcul lui-même, le transcendant mathématicien, ne se donna même pas la peine de prendre une lunette et de regarder dans le ciel si la planète y était réellement! Je crois même qu'il ne l'avait jamais vue... Pour lui, du reste, déjà, et toujours, jusqu'à la fin de sa vie, l'astronomie était tout entière enfermée dans les formules: les astres n'étaient que des centres de force. [...] La connaissance essentielle de l'univers consistait pour lui en équations, en formules, en séries de logarithmes, ayant pour objet la théorie mathématique des vitesses et des forces. ${ }^{40}$

Néanmoins, Flammarion ne peut se contenter de louer Le Verrier, qu'il considère comme le chantre d'une astronomie qui se détourne de la vue du ciel pour se consacrer seulement au chiffre. En fin de compte, il ne fait que « révéle[r] » un « horizon », c'est-àdire qu'il se contente d'ouvrir un espace à explorer. Dans le récit fictionnel Uranie, la muse de l'astronomie ne dit-elle pas à Flammarion, après avoir cité Le Verrier, entre autres, que "[l]'astronomie mathématique va faire place à l'astronomie physique, à la véritable étude de la nature $»^{41}$ ? En somme, c'est un combat des «images contre les nombres $\aleph^{42}$, pour reprendre une expression de Danielle Chaperon, que Flammarion mène contre son ancien directeur. Dans le cadre d'une telle confrontation, la figure de l'astronome mathématicien est placée dans un équilibre instable entre des descriptions trop élogieuses et de pures accusations de myopie.

Le texte met en scène une figure de savant à la fois supérieur, et en même temps décrit sur le modèle topique du scientifique coupé du monde et obnubilé par sa seule spécialité. Force est de constater qu'il frise le ridicule, à l'instar des savants des romans de Jules $V e^{2} e^{43}$. Cette description ambiguë signale un paradoxe dont la gravure de Paul Fouché, intitulée "Le Verrier découvrant Neptune », qui orne le chapitre neuf, rend un compte éloquent ${ }^{44}$. 


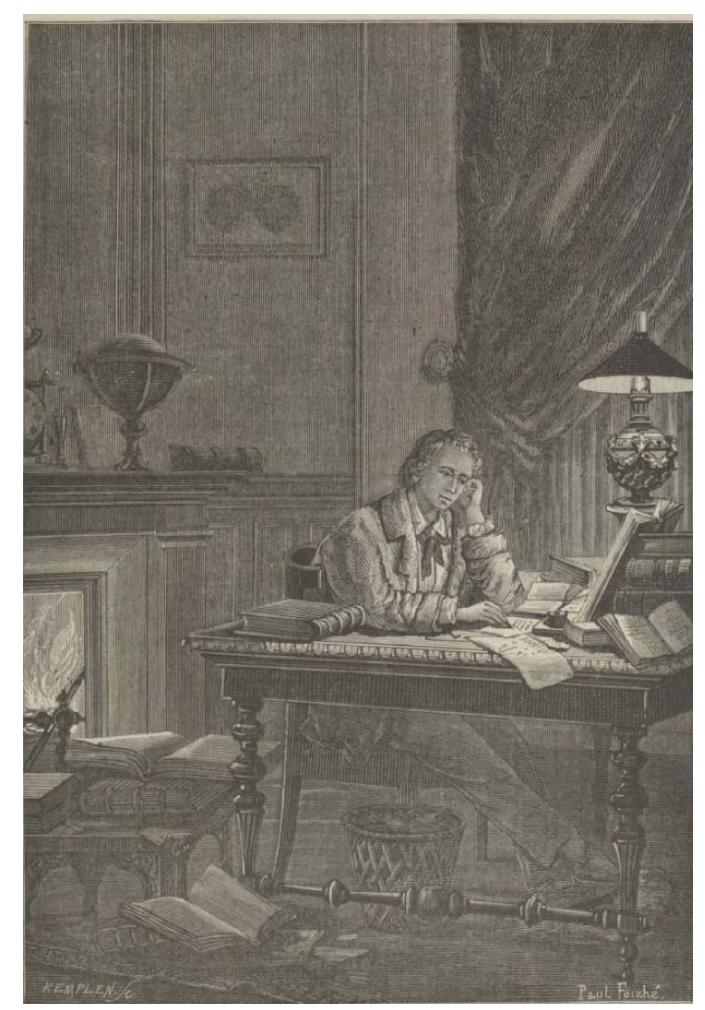

Fig. Frederick Kemplen, Paul Fouché, Le Verrier découvrant la planète Neptune, Gallica, Camille Flammarion, Astronomie populaire, éd. 1881, Sciences et Techniques, 4-V-1286, p. 585

Gallica, BnF, lien hypertexte : http://gallica.bnf.fr/ark:/12148/bpt6k96291926/f612)

Le dessinateur, en effet, représente le savant comme un pur homme de papier. Il est assis à sa table, la nuit, la tête posée sur sa main gauche tandis que la droite, qui tient la plume, écrit des formules sur des pages volantes. Il ressemble alors à un écrivain romantique inspiré. Son inspiration se lit d'abord dans son regard qui fixe un vide plutôt que ce qu'il écrit. Il semble regarder en lui, ou bien regarder simplement le livre ouvert posé devant lui sur un pupitre composé d'autres ouvrages. Figure du progrès cumulatif de l'astronomie, car les progrès dans cette science se fondent sur des observations antérieures reprises et interprétées, ce pupitre de livres, ainsi que la multitude des écrits de diverses formes ouverts un peu partout dans la pièce, sont le signe d'une érudition toute théorique. Si ces divers livres représentent probablement les recueils des " 19 observations anciennes [...] avant 1781 et les 179 observations faites de 1781 à $1845 »^{45}$, ils prouvent que Le Verrier ne se base pas sur l'observation immédiate du ciel mais sur des comptes rendus étrangers, qui intercalent de l'épaisseur entre le ciel et l'astronome. Ce savant est un métempirique : ses livres et son esprit qui fait office de table de calcul (selon qu'il regarde ceux-là ou fixe le vide) sont ses seuls outils de travail. Contrairement à la représentation topique de l'astronome à l'œuvre, Le Verrier est enfermé dans une pièce close. Les lignes de composition sont d'une droiture exemplairement raide et se croisent perpendiculairement, ce qui accentue l'exiguïté du lieu. Il y a bien une fenêtre, mais elle est coupée aux deux tiers par le bord droit de la gravure. De surcroît, elle est recouverte ostensiblement d'un lourd rideau de toile épaisse. Tout concourt à prévenir un contact immédiat entre le ciel et le savant au travail. La seule lumière qui vient éclairer le bureau et la tête du mathématicien, où la résolution du problème est en train d'advenir, ne vient ni du scintillement des étoiles, ni du reflet de la lune, mais d'une lampe à pétrole de style 
Louis XVI. Il est accoudé à une table de même style. La lumière qui jette le jour sur son opération lui vient donc symboliquement des siècles derniers tout comme les assises sur lesquelles il fonde son calcul : les observations qu'il utilise remontent au $\mathrm{XVIII}^{\mathrm{e}}$ siècle et les théories newtoniennes au XVII ${ }^{\mathrm{e}}$ siècle. Si l'on devine des instruments astronomiques, sur et devant la cheminée, ceux-ci sont en arrière-plan, dans la pénombre absolue. Le Verrier, par ailleurs, leur tourne le dos. La posture du savant révèle donc une activité scientifique tout entière tournée vers le calcul et l'hypothèse, au mépris de l'expérience empirique. Le double trait qui caractérise le mathématicien, selon Flammarion, est donc illustré ici : une description superbe et romantique, au premier abord, qui entre en contradiction avec le positivisme de la méthode de Le Verrier, et, dans un second temps, le caractère ridiculement paradoxal de cet astronome que tout concourt à éloigner du ciel. En somme, il s'agit d'une double dégradation. Si Le Verrier fonde les bases d'une astronomie nouvelle, la gravure semble indiquer qu'il est résolument tourné vers le passé. C'est ce que répètera d'ailleurs Uranie, qui place sur le même plan Copernic, Newton et Le Verrier, et les oppose à une astronomie à venir qui serait plus "physique». Comme l'indique la muse de l'astronomie : «Ce sont là d'immenses travaux, des labeurs dignes d'admiration et de transcendantes œuvres, qui mettent en lumière les plus hautes facultés de l'esprit humain. Mais c'est l'armée du passé. Mathématiciens et géomètres. $»^{46}$

Le poète en Flammarion ne peut donc en rester là dans son chapitre sur Neptune. Le disciple de Fontenelle et de ses Entretiens sur la pluralité des mondes habités, en 1889, dans Uranie, qui s'ouvre sur des éléments autobiographiques, relate ses rapports problématiques avec le découvreur de la planète troublante. Encore jeune (le texte s'ouvre ainsi : «J'avais dix-sept ans ${ }^{47}$. Elle s'appelait Uranie $»^{48}$ ), Flammarion est renvoyé de l'observatoire de Paris par son directeur, Le Verrier, "point artiste du tout »" , qui jugeait que l'esprit de l'astronome en herbe avait une tournure trop poétique. Et pour cause : devant une statuette représentant la muse de l'astronomie, le voilà qui se met à rêver: "Chaque fois que j'étais appelé auprès de l'éminent mathématicien, ce n'était point sa gloire universelle qui m'impressionnait le plus. J'oubliais les formules de logarithmes et même l'immortelle découverte de la planète Neptune pour subir le charme de l'œuvre de Pradier. $\aleph^{50}$ Il semble qu'en 1889, dix ans après l'Astronomie populaire, Camille Flammarion confesse son attrait plus grand pour la rêverie que pour le calcul. Certes, la découverte de Neptune est «immortelle», mais le «charme» de l'allégorie l'emporte. Le texte d'Uranie remet en cause l'attraction qu'exerce sur lui la découverte purement algébrique de Le Verrier.

Le chapitre neuf du livre quatrième de l'Astronomie populaire, révélait déjà un attrait qui dépassait la découverte purement algébrique de Le Verrier ${ }^{51}$. En effet, Flammarion prolonge les conséquences du calcul : là où Le Verrier ne daigna pas même jeter un œil à Neptune, Flammarion propose de s'y transporter; il cherche à sonder ce nouvel « horizon ». Il présente dans un premier temps une description matérielle du nouvel objet céleste: il évoque son aspect général, sa distance par rapport au soleil, son positionnement dans le ciel pour un œil terrestre, son diamètre, sa période de rotation et sa composition chimique. Enfin, l'« astronome littérateur » met en place un décentrement du point de vue qui fait basculer le texte dans la fiction:

À cette distance du Soleil, l'astre du jour, s'il peut encore porter ce titre, est réduit de 30 fois en diamètre, de 900 fois en surface et en intensité lumineuse et calorifique; il ne mesure plus que 64" de diamètre. Qu'est-ce que cette lumière et que cette chaleur ? Sans doute ce n'est pas tout à fait une étoile, car le diamètre de la plus brillante étoile, de Sirius, n'est même pas de un centième de seconde, et par 
conséquent le soleil neptunien brille encore comme plus de quarante millions d'étoiles de première grandeur. Mais sortir de la Terre pour aller sur Neptune, c'est quitter la chaleur et la lumière pour pénétrer dans les glaces et dans les ténèbres. ${ }^{52}$ découverts dans les "profondeurs océaniques». Des «êtres charmants, délicats », y vivent apparemment heureux, à hauteur desquels Flammarion focalise son texte selon un nouveau point de vue : «ils préfèrent leur chaumière, leur chaumière obscure au fond des eaux, à peine éclairée de l'éclat de leur phosphorescent amour, et pour eux c'est là le vrai milieu, c'est là le vrai bonheur. ${ }^{53}$ Ce décentrement lui permet de décrire une vie agréable dans un endroit inadapté à la survie humaine. Flammarion rappelle ainsi que le milieu de vie in-forme l'être qui y évolue. Cette loi biologique, héritée du transformisme, vient à l'aide du calcul pour suggérer la possibilité fantaisiste mais plausible d'une vie neptunienne : «Il peut exister des mondes où l'on ne dorme jamais, comme il peut en exister où l'on dorme toujours. C'est peut-être le cas de Neptune. ${ }^{54}$ Presque vingt ans plus tard, Flammarion répond à feu Urbain Le Verrier, qui l'avait précisément renvoyé de l'Observatoire pour avoir défendu ce genre de théories invérifiables (ce que Flammarion

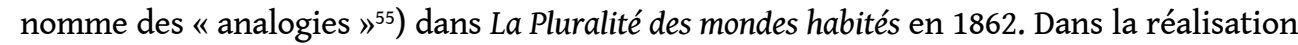
fictionnelle de cette hypothèse, c'est à nouveau le recours au chiffre qui va permettre à la machine optique de Flammarion de se mettre en branle et de "faire voir » à quoi ressemble le quotidien d'un neptunien :

Là, une seule année dure 164 des nôtres ; un enfant de dix ans y a vécu 1640 années terrestres ; une jeune fille de dix-huit ans y épouse, à l'âge de 2950 ans terrestres, le "jeune homme» de ses rêves, âgé lui-même de plus de trois mille ans; et un général en retraite doit être né il y a treize mille ans... [...]

Il va sans dire que là-bas la Terre est complètement invisible, ainsi que Mercure, Vénus et Jupiter. Saturne est une petite étoile qui s'éloigne jusqu'à $18^{\circ}$ du soleil. Pour les neptuniens, le système solaire parait se composer essentiellement du Soleil, de Saturne, d'Uranus, de leur propre monde et de la planète qui, sans doute, gravite au-delà de Neptune. Ces êtres doivent avoir une excellente vue, car elle s'est formée dans un milieu 900 fois moins éclairé que le nôtre : ils doivent apercevoir les étoiles de jour comme de nuit, si l'état de leur atmosphère le leur permet, et leur énorme base d'opération, trente fois supérieure à la nôtre, doit leur avoir permis de calculer longtemps avant nous et beaucoup mieux les parallaxes et les distances des étoiles. ${ }^{56}$

Flammarion décrit ensuite, toujours sur la base de savoirs algébriques, le monde tel que cet autochtone le perçoit. Par exemple, c'est le calcul mathématique qui a permis, plus haut dans le chapitre, de déterminer l'immense masse de Neptune. Il permet à présent de supposer, à partir du volume de Neptune, que les calculs astronomiques de ses hôtes sont plus justes que ceux des Terriens. En effet, les calculs astronomiques reposent le plus souvent sur la trigonométrie. Plus la base que l'on maîtrise effectivement, la base 
terrestre (ou neptunienne) qui représente le côté adjacent dans le calcul trigonométrique, est vaste, et plus le résultat de l'opération sera juste et précis. Le chiffre, donc, permet de voir tout un monde et d'en formuler les représentations hypothétiques mais mathématiquement plausibles. Le recours à l'algèbre comme argument producteur de fiction est en fin de compte une garantie scientifique qui justifie l'imaginaire le plus débridé, quoi qu'en pense le directeur " point artiste du tout » de l'observatoire de Paris.

Flammarion, encore une fois, ne s'arrête pas là. À la projection spatiale il ajoute la projection temporelle. L'esprit de progrès qui le caractérise le pousse à poursuivre plus avant, dans les profondeurs du ciel et du temps, l'enquête optique de l'algèbre : ce n'est pas pour rien que le chapitre dont il est question s'intitule «La planète Neptune et les frontières du domaine solaire ».

De ce que Neptune est la dernière planète que nous connaissions, on n'a aucunement le droit d'en conclure qu'il n'y en a pas d'autres au-delà :

Croire tout découvert est une erreur profonde :

C'est prendre l'horizon pour les bornes du monde.

Nous pouvons même ne pas désespérer de trouver prochainement la première, lorsque les observations de Neptune s'étendront sur un espace assez grand pour que, son orbite étant rigoureusement calculée, les perturbations exercées par la planète extérieure se manifestent d'une manière sensible. [...]

Telle est la dernière étape de notre voyage planétaire, telle est la dernière station du vaste empire du soleil. ${ }^{57}$

29 Le chiffre est présenté comme un conquérant prêt à faire reculer les frontières $d u$ système solaire en recommençant exactement la même opération algébrique. Mais c'est un conquérant qui n'est qu'un éclaireur, et grâce à lui on pourra avoir une approche « sensible » de la dernière planète du système solaire. De fait, comme le note Jean-Claude Pecker dans sa " Postface pour aider à la lecture de l'Astronomie populaire », Flammarion a raison de postuler "l'existence d'une planète transneptunienne responsable des perturbations de Neptune $\aleph^{58}$. Ce que Pecker ne dit pas, en revanche, c'est que la planète Pluton ne sera pas découverte selon le même processus, et qu'en cela la prédiction de Flammarion ne se réalisera pas intégralement.

Ce chapitre, qui clôt le livre quatrième dévolu aux «mondes planétaires ", se termine en évoquant la notion d'unité :

L'unité d'origine n'est pas douteuse, et depuis longtemps même on est autorisé à penser que l'hydrogène est le corps qui se rapproche le plus de la substance primitive essentiellement simple. L'analyse spectrale confirme aujourd'hui ces vues. Les différences entre les planètes de notre grande famille solaire ne sont donc pas essentielles, ce sont surtout des différences de degrés. Nous verrons aussi bientôt que l'analyse chimique des aérolithes et des étoiles filantes appuie cette considération. Les étoiles, soleils de l'espace, sont elles-mêmes sœurs de notre Soleil. Unité d'origine, unité de force, unité de substance, unité de lumière, unité de vie dans l'univers immense, à travers la variété infinie des aspects et des générations. ${ }^{59}$

31 L'univers tout entier est donc placé sous ce signe d'unité dans « la variété ». À partir du moment où tout objet sidéral partage les caractéristiques des objets qui nous sont connus parce qu'ils sont plus proches, le chiffre se contentera d'en désigner l'emplacement. L'approche de ces objets, constitués des mêmes matières que celles que nous expérimentons sensiblement dans notre environnement, se fera par la méthode empirique : nous saurons les voir. 

plus spectaculaires de l'histoire des sciences. Flammarion en explique les étapes point par point. Néanmoins, la résolution de ce problème sert un propos complexe : alors, on l'a vu, que la découverte de Le Verrier entérine le nouveau pouvoir optique du nombre dans la modernité astronomique, Flammarion, défenseur d'une approche plus sensible du ciel, détourne cette découverte pour la récupérer au profit d'une appréhension plus romantique. Il se sert du medium littéraire et des attributs de la fiction pour doter d'une forme spectaculaire un épisode majeur de l'histoire de l'astronomie qu'il réécrit, épisode qu'il contribue à faire entrer dans le mythe scientifique en faisant de Le Verrier, bien malgré lui, un prophète romantique. Si, dans un premier temps, le texte formule un éloge à la gloire de Le Verrier, force est de constater que la seconde partie du chapitre en dépasse, et de loin, les leçons toutes métempiriques. En retour, il semble possible de questionner la véracité de cet éloge. Sous la caresse épidictique se cache en fait la dénonciation d'une science du ciel qui en vient à se dessécher à force de calcul.

La structure du texte porte une leçon qui pousse à prendre acte de la révolution épistémique, justement mise en scène dans ce chapitre, mais à ne pas s'y arrêter. La relation ancillaire qui unit la science à la littérature est donc à double sens. Certes, la littérature est en voie d'extinction, et les sciences seules pourront la sauver. Cependant, ce texte de Flammarion semble évoquer également un danger qui menace les sciences elles-mêmes : celui de l'omniprésence et de l'omnipotence du nombre qui s'est substitué à l'œil comme nouvelle façon de voir. Flammarion accepte la leçon que constitue la découverte algébrique de Neptune, mais il considère que le chiffre ne constitue pas une fin en soi. Il est un outil à mettre au service d'une nouvelle façon de voir. Le nombre doit devenir une boussole qui doit indiquer à l'œil où poser son regard. La saisie du monde doit donc demeurer empirique, ce qui est une condition primordiale pour continuer à produire de la vulgarisation. À ce titre, Flammarion complète les données du nombre en imaginant ses conséquences logiques dans des postulations fictionnelles et spectaculaires.

Il y a donc, c'est certain, du « littérateur " en Flammarion. Néanmoins, la conjonction entre celui-là et le «savant»-ce «et» de la formule «littérateur et savant »s'interprète sémantiquement comme une intersection. Ce chapitre peut être appréhendé selon le modèle d'un chiasme éloquent: la forme fictionnelle prend en charge dans un premier temps le contenu scientifique, puis la forme scientifique légitime un contenu fictionnel. Là réside la puissance de la "machine optique» de Flammarion. Sans contradiction, il peut alors dépasser la sécheresse toute mathématique de la vision du ciel de Le Verrier, pour s'envoler, en prenant appui sur les chiffres comme sur un tremplin, dans les astres de la fiction spéculative propre à l'imaginaire scientifique. Ce faisant, son savoir voir astronomique en vient scientifiquement à formuler des fictions dignes de l'imagination la plus débridée.

\section{NOTES}

1. Charles Bouchet, Le Ciel, poésie, Vendôme, Typ. Lemercier \& fils, 1871, p. 18. 
2. François Arago, Astronomie populaire, Euvres posthumes, t. IV, Paris, L. Guérin, T. Morgand, 1867, p. 515.

3. C'est l'astronome Alexis Bouvard qui, entre autres, calcula ces tables, remarqua les anomalies et formula l'hypothèse d'une planète « troublante ».

4. Pour cette histoire de la découverte de Neptune, voir les Recherches sur les mouvements d'Uranus, où Urbain Le Verrier relate l'historique de ses propres découvertes, Paris, Bachelier, 1846, L'Astronomie populaire de François Arago, Paris, Gide et J.Baudry, 1854, t. IV, p. 509-522, Une histoire de l'astronomie de Jean-Pierre Verdet, Paris, Seuil, 1990, p. 232-234, La science contemporaine , t. I, Le XIX siècle [1961], troisième tome de l'Histoire générale des sciences, René Taton (dir.), Paris, Presses Universitaires de France, coll. "Quadrige », 1995, p. 150-151 et l'article en ligne de James Lequeux, «La découverte de Neptune par Le Verrier (1846) », https://www.bibnum.education.fr/ sites/default/files/neptune-analyse.pdf.

5. C'est le comparant d'une analogie galante, par exemple, au dix-septième chapitre du Tour du monde en quatre-vingts jours de Jules Verne [1873], repris dans Les romans de la terre, Paris, Omnibus, 2002, p. 82: "Telle était donc la situation respective de ces deux hommes [i.e. Passepartout et Fix], et au-dessus d'eux Phileas Fogg planait dans sa majestueuse indifférence. Il accomplissait rationnellement son orbite autour du monde, sans s'inquiéter des astéroïdes qui gravitaient autour de lui. Et cependant, dans le voisinage, il y avait - suivant l'expression des astronomes - un astre troublant qui aurait dû produire certaines perturbations sur le cœur de ce gentleman. Mais non! Le charme de Mrs. Aouda n'agissait point, à la grande surprise de Passepartout, et les perturbations, si elles existaient, eussent été plus difficiles à calculer que celles d'Uranus qui ont amené la découverte de Neptune. "

6. Voir Charles Bouchet, Le Ciel, Vendôme, Typ. Lemercier et fils, 1871 ; ou Augustin Babin, Poème astronomique, troisième partie de la Trilogie morale, Paris, Librairie des sciences psychologiques, 1881.

7. Pour une intéressante contextualisation de ce texte, voir Jean-Claude Pecker, « Postface pour aider à la lecture de l'Astronomie populaire » [1976], reprise dans le tome II de Camille Flammarion, L'Astronomie populaire, Paris, Flammarion, coll. "Champs classiques », 2009. Flammarion cherche ouvertement, par le biais de cet ouvrage, à rendre hommage à Arago dont il reprend le titre des cours qui parurent de manière posthume, et que le vulgarisateur souhaite actualiser en y ajoutant les découvertes modernes.

8. Danielle Chaperon, Camille Flammarion, Entre astronomie et littérature, Paris, Imago, 1997, p. 9.

9. Ibid.

10. Ibid.

11. Maxime Du Camp, préface aux Chants modernes, Paris, M. Lévy frères, 1855.

12. Antoine Compagnon, Les cinq paradoxes de la modernité, Paris, Seuil, 1990, partie I du chapitre 2, « La religion du futur : avant-gardes et récits orthodoxes », p. 49-56.

13. Danielle Chaperon, op. cit., p. 9.

14. Camille Flammarion, L'Astronomie populaire [1880], Paris, Flammarion, coll. "Champs classiques », 2009, chapitre IX du livre quatrième « Les Mondes planétaires », p. 230-246.

15. Danielle Chaperon, op. cit., p. 10.

16. François Arago, op. cit., p. 509-522.

17. Camille Flammarion, Astronomie populaire, op. cit., p. 230.

18. Ibid., p. 231.

19. Ibid.

20. Ibid., p. 232.

21. Voir les analyses de Jacques Lévy dans Histoire générale des sciences, René Taton (dir.), op. cit., p. 150.

22. Camille Flammarion, Astronomie populaire, op. cit., p. 232. 
23. Ibid.

24. Ibid., p. 232-233.

25. Ibid., p. 233.

26. Ibid.

27. Ibid.

28. Ibid., p. 232.

29. Ibid., p. 233.

30. Cette relation empirique étant la suivante : $a=0,4+0,3 \times 2^{\text {n-1 }}$, où $a$ est le demi-grand axe de l'orbite exprimé en unités astronomiques (demi-grand axe de l'orbite terrestre, soit 1,496 108 $\mathrm{km})$, et $n$ un des nombres entiers consécutifs.

31. Camille Flammarion, Astronomie populaire, op. cit., p. 233.

32. Ibid.

33. Ibid., p. 233-235.

34. Ibid., p. 235.

35. Ibid., p. 232.

36. Ibid., p. 237.

37. Ibid., p. 235.

38. Ibid., p. 236.

39. Ibid., p. 235.

40. Ibid., p. 236.

41. Camille Flammarion, Uranie, Paris, Le Livre à la carte-Libris édition, 1998, fac-similé de l'édition Paris, C. Marpon et E. Flammarion, 1889, p. 37.

42. Danielle Chaperon, op. cit., p. 16.

43. Voir par exemple le personnage de Jacques Paganel dans Les Enfants du capitaine Grant [1866], ou Thomas Black dans Le Pays des fourrures [1873] ou le cousin Bénédict dans Un capitaine de quinze ans [1878].

44. Voir Fig. 1.

45. Camille Flammarion, Astronomie populaire, op. cit., p. 232-233.

46. Camille Flammarion, Uranie, op. cit., p. 37.

47. Le Verrier le renverra trois ans plus tard, en 1862, après la parution de La Pluralité des mondes habités.

48. Camille Flammarion, Uranie, op. cit., p. 1.

49. Ibid., p. 3.

50. Ibid., p. 5. Pradier est l'auteur de la statuette d'Uranie.

51. En réalité, c'est une attirance que l'on sent poindre dès Les Merveilles célestes, dont le chapitre $\mathrm{x}$ décrit la découverte de Neptune sans même citer Le Verrier, et chante lyriquement la gloire de l'attraction universelle (Camille Flammarion, Les Merveilles célestes [1862], Paris, Librairie Hachette et $\mathrm{C}^{\mathrm{ie}}, 1875$, p. 239-244).

52. Camille Flammarion, Astronomie populaire, op. cit., p. 240.

53. Ibid., p. 241.

54. Ibid., p. 242.

55. Camille Flammarion, La Pluralité des mondes habités [1866], livre II, «Les mondes planétaires », Paris, Librairie académique, Didier et $C^{\mathrm{ie}}, 1868$, p. 74.

56. Camille Flammarion, Astronomie populaire, op. cit., p. 242-243.

57. Ibid., p. 244-245. Les deux vers cités ouvrent le poème d'Antoine-Marin Le Mierre, «L'utilité des découvertes faites dans les sciences et dans les arts sous le règne de Louis XV » [1756], repris dans les CEuvres de A.-M. Le Mierre, t. III, Paris, Maugeret fils, 1810, p. 288-291. 
58. Jean-Claude Pecker, «Postface pour aider à la lecture de l'Astronomie populaire» [1976], in Camille Flammarion, L'Astronomie populaire, t. II, Paris, Flammarion, coll. "Champs classiques », 2009, p. 647.

59. Camille Flammarion, Astronomie populaire, op. cit., p. 245-246.

\section{RÉSUMÉS}

En 1846, Urbain Le Verrier découvre grâce à la seule méthode algébrique, la planète Neptune qu'il ne prendra même pas la peine d'aller contempler empiriquement. Cette date constitue un point de bascule dans l'histoire de l'astronomie: désormais, un nouveau savoir voir marque la modernité de la science des astres, qui repose exclusivement sur le nombre. Lorsque Flammarion, plus de trente ans plus tard, relate cette anecdote, il explicite dans un premier temps cette révolution paradigmatique. Cependant, l'astronome vulgarisateur, ardent défenseur d'une astronomie sensible et ouverte à l'imagination, ne peut pas se contenter d'entériner un tel changement. Le texte de vulgarisation met alors au profit de la science des outils littéraires en vue d'inventer un nouveau savoir voir, qui prolonge effectivement, sur la base du calcul algébrique, le nombre par l'observation et par l'outil spéculatif de l'imagination.

In 1846, Urbain Le Verrier discovered planet Neptune using nothing but algebraic methodology and would not even bother to try and empirically ascertain its existence. This date represents a tipping point in the history of astronomy: from then on, a new way of seeing (savoir voir) marked the modernity of astral science, which solely rested on numbers. When Flammarion narrated that anecdote more than thirty years later, he was the first to spell out this paradigmatic revolution. However, the astronomy popularizer, who was a staunch defender of a sensible and imaginative astronomy, simply could not endorse such a change. The popularizing text applied literary tools to science for the purposes of inventing a new way of seeing (savoir voir), which actually extended, on the basis of algebraic calculation, the laws of numbers by means of observation and imagination, as a speculative tool.

\section{INDEX}

Mots-clés : Flammarion, astronomie, vulgarisation, fiction spéculative, imaginaire scientifique

\section{AUTEUR}

\section{YOHANN RINGUEDÉ}

Université Paris-Est Marne-la-Vallée, LISAA (EA 4120), Université de Bâle 